\title{
Who Likes Extraverts? Re-examining Motivational Tradeoff Effects in Social Perception
}

\author{
Bastian Jaeger ${ }^{1,2}$, Alex L. Jones ${ }^{3}$, Liam Satchell ${ }^{4}$, Christoph Schild ${ }^{5}, \&$ Florian van Leeuwen ${ }^{1}$ \\ ${ }^{1}$ Department of Social Psychology, Tilburg University \\ ${ }^{2}$ Department of Experimental and Applied Psychology, Vrije Universiteit Amsterdam \\ ${ }^{3}$ Department of Psychology, Swansea University \\ ${ }^{4}$ Department of Psychology, University of Winchester \\ ${ }^{5}$ Department of Experimental Psychology and Personality, RPTU Kaiserslautern-Landau
}

Draft version: 22 March 2023

This paper is currently undergoing peer review. Comments are welcome.

\section{Author Note}

We thank Mitch Brown for sharing the original stimuli and for providing feedback and guidance on the study design.

No conflicts of interest declared.

The study designs were approved by the Ethics Review Board of Tilburg University.

All data and analysis scripts are available at the Open Science Framework (https://osf.io/eugd4/).

Correspondence concerning this article should be addressed to Bastian Jaeger, Department of Social Psychology, Tilburg University, P.O. Box 90153, 5000 LE Tilburg, The Netherlands. E-mail: b.jaeger@uvt.nl. 


\begin{abstract}
Recent research has shown that there are large individual differences in impression formation, but they remain poorly understood. One line of research has tried to explain these differences through the lens of motivational tradeoffs: Perceivers form more positive impressions of targets who appear more likely to address their needs. For example, a perceiver with a strong affiliation motive might prefer extraverted-looking others. As the existing evidence for this hypothesis is mixed, we conducted two preregistered replications of three studies. Using the original stimuli and study design (Study 1, $n=273$ ) and two additional stimulus sets and an improved study design (Study 2, $n=367$ ), we do not find that individual differences in (a) affiliative needs, (b) pathogen concern, or (c) sociosexual orientation predict preferences for extraverted-looking others. Bayesian analyses supported this conclusion. Our findings highlight the need for additional research to understand how individual differences shape social perception. Keywords: social perception, impression formation, motivational tradeoffs, extraversion
\end{abstract}




\section{Who Likes Extraverts? Re-examining Motivational Tradeoff Effects in Social Perception}

People rely on the facial appearance of strangers to form impressions of their personality (Todorov et al., 2015). These impressions are formed quickly and spontaneously (Klapper et al., 2016; Willis \& Todorov, 2006) and can be very consequential: Facial impressions have been shown to influence important decisions, such as legal sentencing (Jaeger et al., 2020; Zebrowitz \& McDonald, 1991), financial decision-making (Duarte et al., 2012), and personnel selection (Gomulya et al., 2017; Ling et al., 2019).

To understand how first impressions are formed, a wealth of studies has examined associations between impressions and specific facial cues, such as resemblances to emotion expressions (Said et al., 2009), facial morphology (Stirrat \& Perrett, 2010), and skin texture (Jaeger et al., 2018). These investigations show how various facial features trigger impressions across perceivers. However, recent work highlights that there are also substantial individual differences in impression formation (Hehman et al., 2017, 2019; Xie et al., 2019). Hehman and colleagues (2017) analyzed 700,000 facial impressions to examine how much variance in impressions is explained by (a) which target was judged, (b) which perceiver was providing the judgment, and (c) the interaction between target and perceiver. Overall, target characteristics explained around $20 \%$ of the variance in personality impressions. While perceiver characteristics explained around $10 \%$ of the variance, the largest proportion was explained by the interaction between target and perceiver characteristics (ca. 35\%). These results demonstrate that the same appearance can elicit different impressions across different perceivers. Thus, to understand how first impressions are formed, researchers need to examine the interplay between characteristics of the person being judged and the person forming the judgment.

Despite the apparent importance of target $\times$ perceiver interactions in social perception, previous studies have almost exclusively focused on the role of target characteristics (Hehman et al., 2019). The question of how individual differences shape first impressions has remained relatively unexplored. Perhaps the most extensive set of studies on how target and perceiver characteristics interact to shape social perception was conducted by Brown and Sacco (2016a, 2016b, 2017; Sacco \& Brown, 2018a). Applying the idea of motivational tradeoffs (Sacco et al., 2014; Sacco \& Brown, 2018b), they examined how various perceiver characteristics, such as individual differences in pathogen concern, influence their impressions. Given the theoretical 
importance of individual differences in social perception and the relative paucity of empirical studies on this topic thus far, we aim to replicate and extend three key findings.

\section{Motivational Tradeoffs in Social Perception}

People constantly balance different needs and goals (Huang \& Bargh, 2014; Kenrick et al., 2010). For instance, one fundamental human motive is to socialize with others - often referred to as the "need to belong" (Baumeister \& Leary, 1995). A stronger need to belong is associated with stronger negative reactions to social exclusion and a stronger motivation to seek out contact with others (Leary et al., 2013). However, this general drive to affiliate with others also comes with potential costs. Infectious disease has been a recurrent threat in humans' evolutionary past and interpersonal contact can increase an individual's risk of pathogen exposure (Schaller \& Park, 2011; Tybur \& Lieberman, 2016). As a consequence, people are sensitive to cues that indicate the potential presence of an infectious disease and increased pathogen concern is associated with aversion to physical contact with others (Tybur et al., 2020). Thus, whereas increased affiliative concern should motivate people to seek out social contact, increased pathogen concern should motivate people to do the opposite. This requires people to make tradeoffs between conflicting goals (Huang \& Bargh, 2014; Kenrick et al., 2010). Ultimately, which goal is pursued will depend on which motive is activated more strongly (Sacco et al., 2014).

Brown and Sacco (2016a, 2016b, 2017) applied the lens of motivational tradeoffs to social perception, investigating how chronically activated motives (e.g., individual differences in affiliative needs or pathogen concern) influence perceivers' impressions of targets who appear more or less extraverted. Extraverted individuals are more gregarious and have larger social networks (Pollet et al., 2011). Thus, people with a stronger need to belong should seek out extraverted others because they are more likely to fulfill their affiliative motives. This reasoning also extends to reactions to perceived extraversion. In line with this reasoning, one study showed a positive association between perceivers' need to belong and their preferences for extravertedlooking (vs. introverted-looking) individuals (Brown \& Sacco, 2017). When participants viewed pairs of images of the same targets - one version manipulated to appear extraverted, the other version manipulated to appear introverted - those with a stronger need to belong more often selected the extraverted-looking image as their preferred appearance. That is, people with a 
stronger need to belong formed more positive impressions of others who look extraverted, presumably because extraverted individuals are more likely to satisfy their affiliative needs.

A similar line of reasoning can explain why individual differences in pathogen concern are negatively related to extraversion preferences (Brown \& Sacco, 2016a). As extraverted individuals are a more likely source of infectious disease due to their increased social activity and larger social networks (Leary et al., 2013; Pollet et al., 2011), people who are chronically concerned about pathogen threats may avoid extraverted-looking others. Brown and Sacco (2016b) also found that an unrestricted sociosexual orientation, that is, an increased motivation for casual sex (Penke \& Asendorpf, 2008), was positively related to facial extraversion preferences. As extraverted individuals are more open to not only social, but also sexual relationships (Nettle, 2005), they may seem more approachable to people with an unrestricted sociosexual orientation. Together, these findings not only extend the idea of motivational tradeoffs to the domain of social perception (Sacco \& Brown, 2018b; Young et al., 2021), they also underscore the importance of considering both perceiver characteristics (e.g., pathogen concern) and target characteristics (e.g., perceived extraversion) when trying to understand how first impressions arise (Hehman et al., 2019).

\section{A Critical Examination of the Empirical Evidence}

While the theoretical arguments for the three discussed relations seem persuasive, the available empirical evidence is less strong. In all three studies, impressions of targets varying in perceived extraversion were measured with a two-alternative forced-choice (2AFC) design (Brown \& Sacco, 2016b, 2016a, 2017). Participants viewed 40 pairs of face images and indicated, for each pair, which face they prefer. Each pair contained two images of the same target that was manipulated to vary in perceived extraversion. To create the different face versions, the same face was morphed with a prototypically extraverted-looking face (i.e., the averaged face of individuals with high scores on extraversion) and a prototypically introvertedlooking face (i.e., the averaged face of individuals with low scores on extraversion). This method has been criticized for its poor external validity-people rarely encounter twin faces that only vary on one dimension in everyday life — and, perhaps more importantly, its proneness for producing false positive results (Bovet et al., 2022; DeBruine, 2020; Jones \& Jaeger, 2019; Satchell et al., 2023). The 2AFC design highlights even subtle differences between face pairs and participants may perceive and rely on these differences even though they would go unnoticed in 
everyday conditions. Thus, it is unclear if results obtained with a $2 \mathrm{AFC}$ design would also emerge when people judge unmanipulated images of individuals varying in apparent extraversion (i.e., when they judge others under conditions that more closely resemble everyday impression formation).

In addition to these methodological limitations, the reported results do not lend unequivocal support for the motivational tradeoff hypothesis. In the study that examined the role of individual differences in pathogen concern in impression formation (Brown \& Sacco, 2016a), pathogen concern was measured with the Perceived Vulnerability to Disease (PVD) scale (Duncan et al., 2009), which consists of two subscales: Perceived Infectability (reflecting beliefs about a person's susceptibility to contagious disease) and Germ Aversion (reflecting a person's discomfort and worry with regard to potential pathogen threats). The Germ Aversion scale better captures what is typically referred to as pathogen concern and also correlates more strongly with other measures of pathogen concern, such as pathogen disgust sensitivity (Duncan et al., 2009; Tybur et al., 2014). Thus, we would expect to see a negative correlation between Germ Aversion and extraversion preferences, but not necessarily a negative correlation for Perceived Infectability. Contrary to these predictions, a negative association was only observed for Perceived Infectability (and this association was only "marginally significant"). ${ }^{1}$

Results for the role of individual differences in sociosexual orientation were also mixed. While stronger preferences for extraverted-looking men were expected among more unrestricted women, the predicted three-way interaction did not emerge. A positive correlation between sociosexual orientation and extraversion preferences was observed for male (but not female) targets, and this association did not differ between male and female participants (Brown \& Sacco, 2016b).

The motivational tradeoffs framework offers a valuable perspective on how target and perceiver characteristics might interact in impression formation. Yet, existing evidence in favor of various hypotheses that were derived from this framework is mixed. Some of the unexpected results may point to interesting boundary conditions. However, they may also constitute false

\footnotetext{
${ }^{1}$ Although no gender differences were explicitly hypothesized, follow-up analyses that tested for an association between perceived infectability and extraversion preferences showed a significant effect for male participants rating female targets, a marginally significant effect for female participants rating male targets, and no significant effects for male participants rating male targets or female participants rating female targets.
} 
positives as increasing the number of statistical tests (e.g., by analyzing different subgroups of participants and targets) without adjusting the significance threshold is bound to produce significant results (Simmons et al., 2011). ${ }^{2}$ Stronger tests of these hypotheses are needed.

\section{The Present Studies}

Here, we present the results of two preregistered replication studies, in which we examine the role of three individual difference variables in social perception. In both studies, we test whether perceivers show stronger preferences for extraverted-looking targets if they score higher on the need to belong (Brown \& Sacco, 2017), lower on pathogen concern (Brown \& Sacco, 2016a), and higher on an unrestricted sociosexual orientation (Brown \& Sacco, 2016b). We test these hypotheses with two independent samples of participants and three samples of face stimuli, moving from a relatively close replication (often also referred to as a direct replication) of the original findings to a more conceptual replication that attempts to improve on the original methodology (LeBel et al., 2019; Tierney et al., 2020).

In Study $1(n=273)$, we use the same face stimuli and the same study design used in the original studies. Participants view pairs of images of the same individual that were manipulated to appear more extraverted or more introverted and indicate which face version they prefer. Individual differences in facial extraversion preferences are indexed by how often participants choose the extraverted face version. In Study $2(n=367)$, we conducted two additional tests of the hypotheses. In our first test, we use the same study design, but a different stimulus set (Walker et al., 2018; Walker \& Vetter, 2016) to test whether the original findings generalize to different stimuli. In our second test, we use a different set of face stimuli, taken from the 10k Face Database (Bainbridge, Isola, Blank, et al., 2013), and we rely on a different study design for measuring participants' perceptions. Instead of a 2AFC design with morphed versions of the same face, we present naturalistic face images in a serial fashion and participants rate targets on a Likert scale.

\footnotetext{
${ }^{2}$ Recent large-scale replication projects have offered first insights into the predictors of replicability (Altmejd et al., 2019; Open Science Collaboration, 2015). In these studies, findings with $\mathrm{p}$-values that were just below the significance threshold $(.01<p<.05)$ were less likely to replicate. The results for potential associations between facial extraversion preferences and pathogen concern (Brown \& Sacco, 2016a), and need to belong (Brown \& Sacco, 2017) were both associated with $p$-values that fall within this range, which raises further concerns about the robustness of the findings.
} 
In both studies, we report the results of both frequentist and Bayesian analyses, which also allowed us to quantify evidence in favor of the null (Aczel et al., 2017; Wagenmakers, 2007).

\section{Study 1}

In Study 1, we conducted close replications of three effects. We used the same set of 40 face pairs and the same rating technique (2AFC) that were used in the original studies. We examined whether participants who report lower pathogen concern, higher need to belong, and a more unrestricted sociosexual orientation would form more positive impressions of extravertedlooking targets (Brown \& Sacco, 2016a, 2016b, 2017). We also examined whether the positive correlation between an unrestricted sociosexual orientation and facial extraversion preferences would be larger for women than for men (Brown \& Sacco, 2016b). ${ }^{3}$

\section{Methods}

All data, analysis scripts, and preregistration documents for the current studies are available at the Open Science Framework (https://osf.io/eugd4/). Our preregistrations specify the study design, the planned sample size and stopping rule for data collection, data exclusion criteria, and planned analyses. We explicitly mention any deviation from our preregistered approach. We report all manipulations, measures, and data exclusions.

Participants. Our main goal was to examine associations between facial extraversion preferences and various individual difference measures. An a priori power analysis showed that a sample of 193 participants is needed to detect a small correlation $(r=.20)$ with $80 \%$ power (and $\alpha=5 \%$ ). We therefore aimed to recruit a minimum sample of 193 participants with the final sample being determined by how many participants completed the study in a window of three weeks. Note that this is only an approximation as our analyses focus on the results of multilevel regression models.

A total of 277 participants participated in the study voluntarily. The study was advertised in various social media groups of Dutch universities and participants took part voluntarily. In line with our preregistered exclusion criteria data from 4 participants (1.44\%) who indicated poor

\footnotetext{
${ }^{3} \mathrm{We}$ also preregistered to test for a positive association between participants' extraversion and their facial extraversion preferences (Sacco \& Brown, 2018b). Due to a technical error, participants' extraversion scores were not recorded and we were only able to test this hypothesis in Study 2.
} 
English proficiency were excluded, leaving a final sample of 273 participants $\left(M_{\text {age }}=23.18\right.$ years, $S D_{\text {age }}=6.14 ; 69.60 \%$ female, $29.67 \%$ male, $0.73 \%$ other).

Materials and Procedure. We used the same stimuli and rating technique as in the original studies (Brown \& Sacco, 2016b, 2016a, 2017). Participants viewed 40 pairs of facial photographs. Each pair of photographs showed the face of the same individual. Perceived extraversion was manipulated by morphing the face with prototypically introverted- and extraverted-looking faces (Holtzman, 2011). Each face represented a 50\%/50\% morph of the original face and the prototype. Thus, each pair contained an introverted and an extraverted version of the same face and participants were asked to indicate which version they "prefer" in a two-alternative forced-choice design. We randomized the order in which the face pairs were displayed and whether the introverted- or extraverted-looking face was presented on the left.

Next, participants completed various questionnaires. We measured individual differences in pathogen concern with the 15-item Perceived Vulnerability to Disease Scale (Duncan et al., 2009). The Perceived Infectability subscale $(\alpha=0.86)$ captures beliefs about a person's subjective susceptibility to contagious disease (e.g., "If an illness is 'going around,' I will get it."). The Germ Aversion subscale $(\alpha=0.70)$ captures differences in affective responses to pathogen threats (e.g., "It really bothers me when people sneeze without covering their mouths."). Participants indicated their responses on a seven-point scale ranging from 1 (strongly disagree) to 7 (strongly agree).

We measured individual differences in affiliative needs with the 10-item Need to Belong scale (Leary et al., 2013), which includes items such as "I want other people to accept me". Participants indicated their responses on a seven-point scale ranging from 1 (strongly disagree) to 7 (strongly agree).

We measured individual differences in sociosexual orientation with the 9-item Sociosexual Orientation Inventory-Revised (Penke \& Asendorpf, 2008), which captures preferences for uncommitted vs. committed sexual relationships. ${ }^{4}$ The scale consists of three subscales, which measure sexual behavior ("With how many different partners have you had sex within the past 12 months?"), attitudes towards casual sex (“Sex without love is OK."), and

${ }^{4}$ In line with Brown and Sacco (2016b) and our preregistered analysis plan, we omitted 42 participants who were above the age of 40 or did not identify as heterosexual from analyses examining associations between sociosexual orientation and extraversion preferences. 
sexual desire ("In everyday life, how often do you have spontaneous fantasies about having sex with someone you have just met?"). Participants indicated their responses on nine-point scales, with higher scores indicating a more unrestricted sociosexuality. In line with the original study (Brown \& Sacco, 2016b), we focus on participants' average score across the three subscales.

Descriptive statistics for all measures are presented in Table 1.

Analysis strategy. All analyses were conducted in R (R Core Team, 2021). We estimated multilevel regression models with the lme4 (Bates et al., 2015) and lmerTest package (Kuznetsova et al., 2016). We report the results of Bayesian analyses alongside frequentist statistics. We followed the approach proposed by Wagenmakers (2007) to compute Bayes factors for all critical tests. We estimated models with and without the variable of interest and computed the Bayesian information criterion (BIC), an indicator of model fit, for both models. Comparing the BICs of both models quantifies the extent to which the variable of interest improved model fit. We converted this measure to an approximation of the Bayes factor by using the following formula: $B F_{10} \approx \exp \left(\frac{B I C\left(H_{0}\right)-B I C\left(H_{1}\right)}{2}\right)$, where $\mathrm{BF}_{10}$ represents the Bayes factor in favor of the alternative hypothesis and $\mathrm{BIC}\left(\mathrm{H}_{1}\right)$ and $\mathrm{BIC}\left(\mathrm{H}_{0}\right)$ denote the fit of the models with and without the variable of interests, respectively. For interpretative convenience, we always display Bayes factors so that they reflect support for the favored hypothesis (i.e., $B F_{10}$ when evidence favors the alternative hypothesis and $B F_{01}$ when evidence favors the null hypothesis). Following Jeffreys (1961), we label evidence as anecdotal $(1 \leq B F<3)$, substantial $(3 \leq B F<10)$, strong $(10 \leq B F<$ $30)$, very strong $(30 \leq B F<100)$, or decisive $(B F \geq 100)$.

\section{Results}

We examined whether people generally reported increased liking for extraverted-looking faces. We coded participants' responses as 0 when they preferred the introverted-looking face and as 1 when they preferred the extraverted-looking face. We tested whether extraversion preferences were stronger than expected by chance (i.e., 50\%) by examining the intercept in a multilevel logistic regression model with random intercepts per participant and target. The intercept was not significant and a Bayesian analysis indicated decisive evidence in favor of the null hypothesis, $b=-0.017, S E=0.109, O R=0.98,95 \% \mathrm{CI}[0.79,1.22], p=.873, B F_{01}=103.2$. Participants chose the extraverted-looking face $49.45 \%$ of the time. Thus, in contrast to earlier studies with the same stimuli (Brown \& Sacco, 2016b, 2016a, 2017), participants did not show a preference for extraverted-looking or introverted-looking faces. In line with these earlier studies, 
we did find that extraversion preferences differed for male and female targets. Adding target gender to the model $(0=$ female target, $1=$ male target $)$ yielded a significant effect with decisive evidence in favor of the alternative hypothesis, $b=-0.858, S E=0.157, O R=0.42,95 \%$ CI [0.31, 0.58], $p<.001, B F_{10}=722.9$. When rating male faces, participants chose the extraverted-looking face $39.96 \%$ of the time, but when rating female faces, participants chose the extraverted-looking face $58.94 \%$ of the time.

\section{Table 1}

Descriptive statistics

\begin{tabular}{|c|c|c|c|c|c|c|}
\hline & \multicolumn{3}{|c|}{ Study $1(n=273)$} & \multicolumn{3}{|c|}{ Study $2(n=367)$} \\
\hline & $\alpha$ & $M$ & $S D$ & $\alpha$ & $M$ & $S D$ \\
\hline Male (in \%) & - & 29.67 & - & - & 15.26 & - \\
\hline Age & - & 23.18 & 6.14 & - & 19.90 & 3.70 \\
\hline Extraversion preference (in \%) & - & 49.45 & 13.75 & - & 83.07 & 15.80 \\
\hline Perceived infectability & .86 & 3.36 & 1.16 & .87 & 3.24 & 1.17 \\
\hline Germ aversion & .70 & 4.07 & 1.01 & .74 & 3.76 & 1.07 \\
\hline Need to belong & .75 & 3.22 & 0.58 & .75 & 3.25 & 0.59 \\
\hline Sociosexual orientation & .83 & 3.61 & 1.39 & .84 & 3.37 & 1.35 \\
\hline Extraversion & - & - & - & .82 & 3.27 & 0.69 \\
\hline
\end{tabular}

Note. The mean for "male" denotes the percentage of male participants in the sample. The mean for "extraversion preference" denotes the percentage of times participants chose the extraverted face.

Next, we conducted our critical tests. We examined the associations between facial extraversion preferences and four individual difference measures. Four separate multilevel logistic regression models with random intercepts per face and participant were estimated in which face preference ( $0=$ introverted-looking, $1=$ extraverted-looking) was regressed on the individual difference measures (results for all models are displayed in Figure 1).

First, we examined whether participants scoring higher on pathogen concern showed stronger extraversion preferences. There were no significant associations between extraversion preferences and perceived infectability (with decisive evidence in favor of the null hypothesis), $b$ $=-0.003, S E=0.038, O R=1.00,95 \%$ CI $[0.92,1.07], p=.941, B F_{01}=104.2$ (see Figure 1a), or 
germ aversion (with very strong evidence in favor of the null hypothesis), $b=-0.038, S E=$ $0.038, O R=0.96,95 \%$ CI $[0.89,1.04], p=.326, B F_{01}=64.61$ (see Figure $1 \mathrm{~b}$ ).

We also found no significant association between extraversion preferences and individual differences in need to belong (with very strong evidence in favor of the null hypothesis), $b=$ $0.058, S E=0.038, O R=0.94,95 \%$ CI $[0.88,1.02], p=.129, B F_{01}=33.10$ (see Figure 1c).

In the original study, it was predicted that people with a more unrestricted sociosexual orientation would show stronger extraversion preferences and that this association would be stronger for women rating men (vs. men rating women; Brown \& Sacco, 2016b). We therefore estimated a model in which face preference was regressed on sociosexual orientation (with higher scores indicating a more unrestricted sociosexual orientation $)$, gender $(-0.5=$ women rating men, $0.5=$ men rating women), and their interaction. There was a significant main effect of gender (with strong evidence in favor of the alternative hypothesis), $b=0.743, S E=0.183$, $O R=2.10,95 \% \mathrm{CI}[1.46,3.03], p<.001, B F_{10}=21.94$, showing that men (rating women) showed stronger extraversion preferences than women (rating men). We did not find the predicted main effect of sociosexual orientation (with very strong evidence in favor of the null hypothesis), $b=-0.037, S E=0.055, O R=0.96,95 \% \mathrm{CI}[0.86,1.07], p=.499, B F_{01}=54.10$, or an interaction effect between sociosexual orientation and gender (with very strong evidence in favor of the null hypothesis), $b=-0.065, S E=0.110, O R=0.94,95 \%$ CI $[0.75,1.16], p=.556$, $B F_{01}=57.19$ (see Figure 1d). 


\section{Figure 1}

Associations between facial extraversion preferences and individual differences in (a) perceived infectability, (b) germ aversion, $(c)$ need to belong, and $(d)$ sociosexual orientation (the graph displays results for the predicted interaction effect with participants' gender; Study 1)
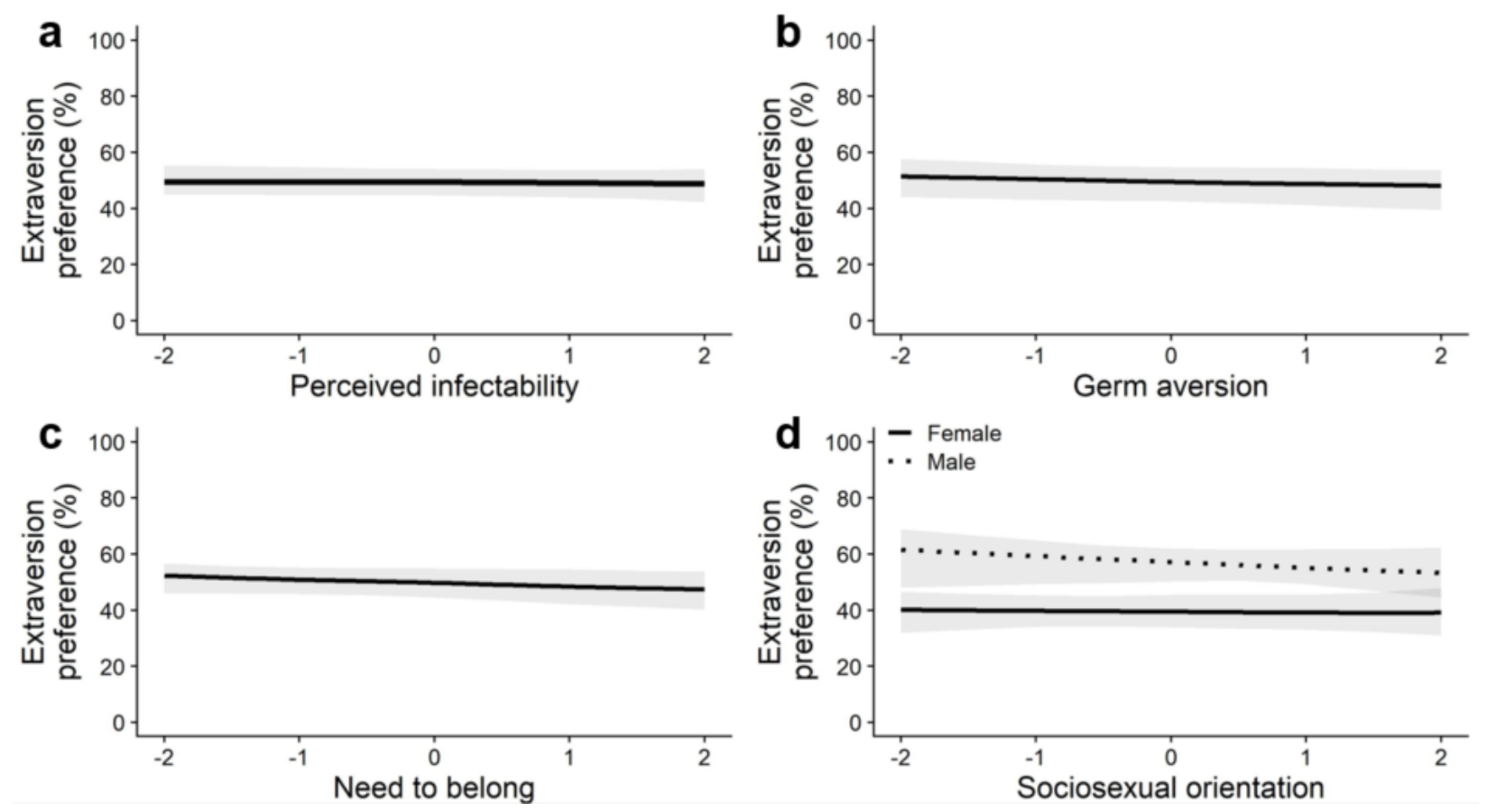

Note. All individual difference measures were $z$-standardized.

\section{Discussion}

In Study 1, we conducted a relatively close replication of three studies that examined the role of individual differences in social perception (Brown \& Sacco, 2016b, 2016a, 2017). Using the same face stimuli and the same study design, we tested whether participants who score low on pathogen concern, participants who score high on need to belong, and participants who report a more unrestricted sociosexual orientation show a stronger preference for extraverted-looking others. We did not find support for any of the three hypotheses and Bayesian analyses showed strong to decisive evidence in favor of the null hypothesis.

\section{Study 2}

In Study 2, we conducted two additional replication attempts. We tested the same three hypotheses but measured facial extraversion preferences in two alternative ways. First, we employed the same rating method as in Study 1 (and the original studies), but we used a different set of stimuli. Participants indicated their impressions for 40 face pairs, taken from the Basel 
Face Database (Walker et al., 2018; Walker \& Vetter, 2016), that contrasted two versions of the same face varying in apparent extraversion.

Second, given recent critiques of the 2AFC method (DeBruine, 2020; Jones \& Jaeger, 2019; Satchell et al., 2023), we tested our hypotheses using an alternative rating method and a third set of face stimuli. Participants viewed 100 faces taken from the 10k Faces Database, which contains face images scraped from the internet (Bainbridge, Isola, Blank, et al., 2013).

Participants viewed targets in a serial fashion and indicated their impressions on a Likert scale. Rather than indicating their general preference for different targets, we asked participants to indicate how much they think they would like the person. The database contains extensive validation data, which also includes average ratings of targets' perceived extraversion. This allowed us to test whether people generally liked extraverted-looking targets. More importantly, this also allowed us to test whether the likeability of targets varying in apparent extraversion is predicted by participants' pathogen concern, need to belong, and sociosexual orientation.

We also considered the role of another trait in explaining facial extraversion preferences. People generally have favorable impressions of others with a similar personality (Montoya et al., 2008; Youyou et al., 2017). This may also extend to targets' perceived personality. Thus, extraverted people may show an increased preference for extraverted-looking others. An initial study did not find support for this prediction (Sacco \& Brown, 2018b). In the present study, we conducted an additional test of the hypothesis.

\section{Methods}

Participants. Our main goal was to examine associations between facial extraversion preferences and various individual difference measures. An a priori power analysis showed that a sample of 319 participants is needed to detect a small correlation $(r=.20)$ with $95 \%$ power (and $\alpha=5 \%$ ). We therefore aimed to recruit a minimum sample of 319 participants with the final sample being determined by how many participants completed the study in a window of three weeks. Note that this is only an approximation as our analyses focus on the results of multilevel regression models.

We recruited 390 first-year psychology students from a Dutch university who completed the study in return for partial course credit. In line with our preregistered exclusion criteria, data from 5 participants (1.28\%) who indicated poor English proficiency and from 18 participants 
(4.68\%) who completed the study on a cell phone were excluded, leaving a final sample of 367 participants $\left(M_{\text {age }}=19.90\right.$ years, $S D_{\text {age }}=3.70 ; 83.11 \%$ female, $15.26 \%$ male, $1.63 \%$ other $)$.

Materials and Procedure. Participants completed two rating tasks that measured their preferences for extraverted-looking others. First, participants viewed 40 pairs of facial photographs taken from the Basel Face Database (Walker et al., 2018; Walker \& Vetter, 2016). Each pair showed the face of the same individual. Perceived extraversion was manipulated by morphing the face with prototypically introverted and extraverted faces. Thus, each pair contained an introverted and extraverted face version and participants were asked to indicate which face version they "prefer" in a two-alternative forced-choice design (in line with the original studies; Brown \& Sacco, 2016a, 2016b, 2017; Sacco \& Brown, 2018a). We randomized the order in which the face pairs were displayed and whether the introverted- or extravertedlooking face was presented on the left.

Participants then completed an unrelated questionnaire (measuring perceptions of animals) for approximately 3 minutes before commencing with the second rating task. Participants viewed 100 faces, which were randomly sampled from the 10k Faces Database (Bainbridge, Isola, Blank, et al., 2013; Bainbridge, Isola, \& Oliva, 2013). The database includes ratings on a wide range of dimensions, including sociability and introversion. These ratings were provide by 30 workers recruited from Amazon Mechanical Turk who rated each face on a ninepoint scale. Individual ratings were then averaged to create an average score for each face (Bainbridge, Isola, \& Oliva, 2013). We reverse-scored introversion ratings and averaged them with sociability ratings to create a measure of perceived extraversion (the correlation between ratings on the two dimensions was $r=.60$ ). Photographs were presented sequentially in a random order and participants indicated, on a nine-point scale, how much they think they would like the person in photo on a scale that ranged from 1 (not at all) to 9 (extremely).

We examined the association between facial extraversion preferences and five individual difference measures: perceived infectability $(\alpha=0.87)$ and germ aversion $(\alpha=0.74$; Duncan et al., 2009), need to belong ( $\alpha=0.75$; Leary et al., 2013), sociosexuality ( $\alpha=0.84$; Penke $\&$ Asendorpf, 2008) $)^{5}$, and extraversion $(\alpha=0.82)$. The first four constructs were measured with the

\footnotetext{
${ }^{5}$ In line with Brown and Sacco (2016b) and our preregistered analysis plan, we omitted 78 participants who were above the age of 40 or did not identify as heterosexual from analyses examining associations between sociosexual orientation and extraversion preferences.
} 
same scales as in Study 1. We measured individual differences in extraversion with the 10-item Extraversion subscale of the HEXACO inventory (Ashton \& Lee, 2009). Participants indicated their responses on a seven-point scale ranging from 1 (strongly disagree) to 5 (strongly agree).

Descriptive statistics for all measures are presented in Table 1.

Analysis strategy. We followed the same analysis strategy as in Study 1. For all primary tests, we report the results of frequentist and Bayesian analyses.

\section{Results}

Two-alternative forced-choice design (Basel faces). First, we analyzed participants' responses when rating the face stimuli from the Basel Face Database. We coded participants' responses as 0 when they preferred the introverted-looking face and as 1 when they preferred the extraverted-looking face. Participants chose the extraverted-looking face $83.07 \%$ of the time. We tested whether this preference was stronger than expected by chance (i.e., 50\%) by examining the intercept in a multilevel logistic regression model with random intercepts per participant and target. This yielded a significant intercept and decisive evidence in favor of the alternative hypothesis, $b=2.221, S E=0.148, O R=9.21,95 \% \mathrm{CI}[7.06,12.03], p<.001, B F_{10}=1.341 \times$ $10^{16}$. Thus, participants generally preferred extraverted-looking faces. We also tested if this preference was different for male and female targets. Adding target gender to the model $(0=$ female target, $1=$ male target) did not yield a significant effect with decisive evidence in favor of the null hypothesis, $b=0.036, S E=0.260, O R=1.04,95 \%$ CI $[0.62,1.67], p=.889, B F_{01}=$ 120.0. When rating male faces, participants chose the extraverted-looking face $82.76 \%$ of the time, but when rating female faces, participants chose the extraverted-looking face $83.32 \%$ of the time.

Next, we conducted our critical tests. We examined associations between facial extraversion preferences and the five individual difference measures. Five separate multilevel logistic regression models with random intercepts per face and participant were estimated in which face preference ( 0 = introverted-looking, 1 = extraverted-looking) was regressed on each individual difference measure (results for all models are displayed in Figure 2).

First, we examined whether participants scoring higher on pathogen concern showed weaker extraversion preferences. There was a significant negative association between extraversion preferences and perceived infectability, $b=-0.175, S E=0.074, O R=0.84,95 \% \mathrm{CI}$ $[0.71,0.99], p=.018, B F_{01}=7.70$ (see Figure 2a). Preferences for extraverted-looking 
individuals were weaker among people who scored higher on perceived infectability, but this effect was very small. A one standard deviation increase in perceived infectability was associated with a 1.47 percentage point decrease in extraversion preferences and a Bayesian analysis indicated substantial evidence in favor of the null hypothesis. The association between extraversion preferences and germ aversion was not significant (with very strong evidence in favor of the null hypothesis), $b=0.073, S E=0.075, O R=1.08,95 \%$ CI $[0.93,1.25], p=.326$, $B F_{01}=75.20$ (see Figure $2 b$ ).

We did not find a significant association between extraversion preferences and individual differences in need to belong (with very strong evidence in favor of the null hypothesis), $b=$ 0.077, $S E=0.074, O R=1.08,95 \%$ CI $[0.92,1.24], p=.298, B F_{01}=71.07$ (see Figure 2c).

In the original study, it was predicted that people with a more unrestricted sociosexual orientation would show stronger extraversion preferences and that this association would be stronger for women rating men (vs. men rating women; Brown \& Sacco, 2016b). We therefore estimated a model in which face preference was regressed on sociosexual orientation (with larger scores indicating a more unrestricted sociosexual orientation), gender $(-0.5=$ men rating women, $0.5=$ women rating men), and their interaction. As predicted, there was a significant positive effect of sociosexual orientation, $b=0.420, S E=0.139, O R=1.52,95 \%$ CI $[1.14,2.09], p=$ $.002, B F_{10}=1.27$. Participants with a more unrestricted sociosexual orientation showed stronger preferences for extraverted-looking individuals of the opposite sex. However, a Bayesian analysis only yielded anecdotal evidence in favor of this effect. The main effect of gender was marginally significant (with strong evidence in favor of the null hypothesis), $b=-0.736, S E=$ $0.408, O R=0.48,95 \% \mathrm{CI}[0.22,1.15], p=.071, B F_{01}=15.20$. There was also a significant interaction effect between sociosexual orientation and gender, $b=0.571, S E=0.278, O R=1.77$, $95 \%$ CI $[1.05,3.13], p=.040, B F_{01}=8.90$ (see Figure 2d). However, Bayesian analyses yielded very strong evidence in favor of the null hypothesis and the observed pattern was opposite of what was found in the original study. The positive association between a more unrestricted sociosexual orientation and extraversion preferences was stronger for men rating women (vs. women rating men).

Finally, we found a positive effect of participants' extraversion on their facial extraversion preferences (with decisive evidence in favor of the alternative hypothesis), $b=$ $0.356, S E=0.071, O R=1.43,95 \%$ CI $[1.25,1.66], p<.001, B F_{10}=1287.9$ (see Figure $\left.2 \mathrm{e}\right)$. A 
one standard deviation increase in extraversion was associated with a 2.69 percentage point increase in extraversion preferences. In other words, more extraverted participants showed more positive impressions of extraverted-looking targets.

\section{Figure 2}

Associations between facial extraversion preferences and individual differences in (a) perceived infectability, (b) germ aversion, (c) need to belong, (d) sociosexual orientation (the graph displays results for the predicted interaction effect with participants' gender), and (e) extraversion (Study 2)
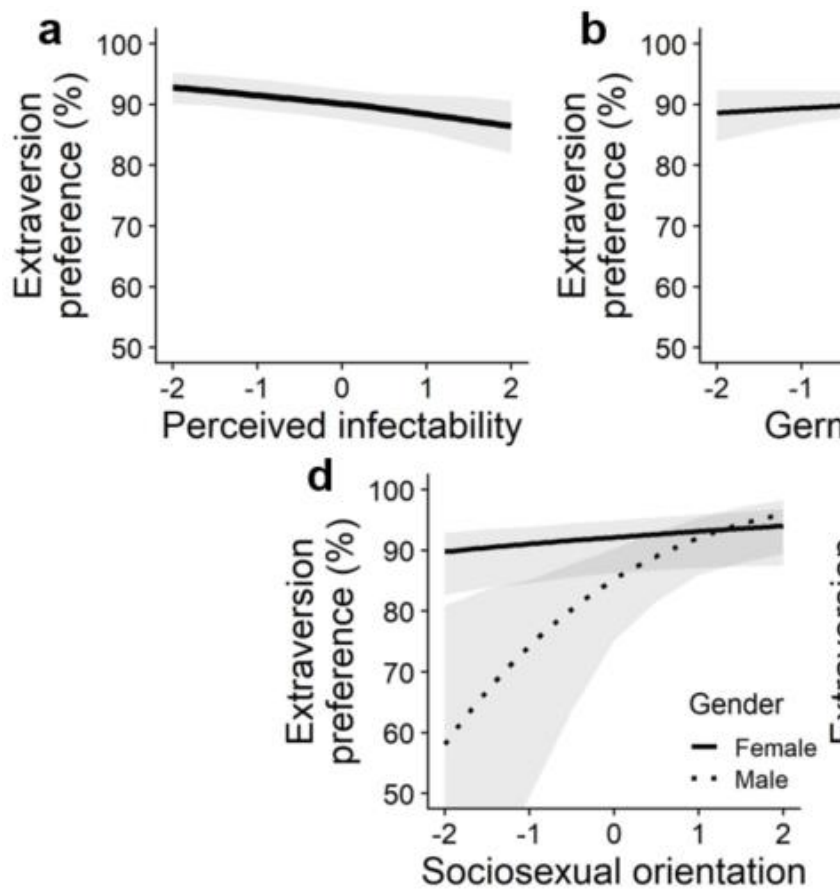
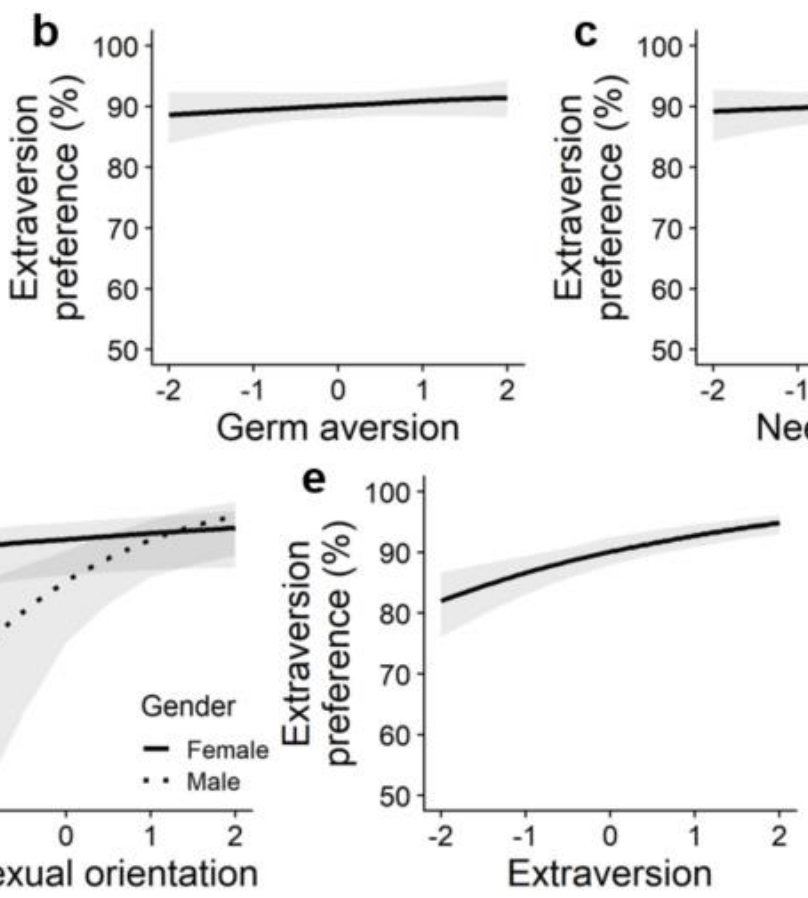

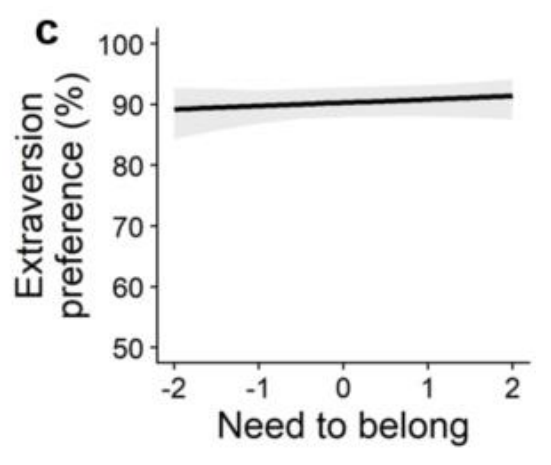

Note. All individual difference measures were $z$-standardized.

Continuous ratings (10k faces). Next, we analyzed participants' ratings of the face stimuli taken from the 10k Faces Database. We examined whether people reported an increased liking for more extraverted-looking targets. We regressed likeability ratings on the apparent extraversion of targets in a multilevel linear regression model with random intercepts per participant and target. This yielded a significant positive effect and decisive evidence in favor of the alternative hypothesis, $b=0.649, S E=0.087,95 \% \mathrm{CI}[0.495,0.812], p<.001, B F_{10}=4.42 \times$ $10^{6}$. Thus, participants generally rated more extraverted-looking targets as more likeable. We 
again tested whether the gender of targets moderates this effect. Adding target gender to the model ( 0 = female target, $1=$ male target $)$ did not yield a significant interaction effect between target gender and apparent extraversion with decisive evidence in favor of the null hypothesis, $b$ $=0.209, S E=0.207,95 \%$ CI $[-0.210,0.619], p=.315, B F_{01}=221.73$.

We then examined associations between facial extraversion preferences and the five individual difference measures. Five separate multilevel linear regression models with random intercepts per face and participant were estimated. We regressed likeability ratings on apparent extraversion, each individual difference measure of interest, and their interaction (results for all models are displayed in Figure 3). The interaction effects represent the crucial tests of our hypotheses, as they show whether associations between targets' apparent extraversion and participants' likeability ratings were moderated by our variables of interest.

First, we examined whether participants scoring higher on pathogen concern showed weaker extraversion preferences. The interaction effect between apparent extraversion and perceived infectability was not significant (with decisive evidence in favor of the null hypothesis), $b=-0.003, S E=0.009,95 \%$ CI $[-0.019,0.014], p=.775, B F_{01}=8101$ (see Figure $3 a)$, and neither was the interaction effect between apparent extraversion and germ aversion (with decisive evidence in favor of the null hypothesis), $b=0.013, S E=0.009,95 \% \mathrm{CI}[-0.005$, 0.031], $p=.148, B F_{01}=2963$ (see Figure 3b).

There was a significant interaction effect between apparent extraversion and need to belong, $b=0.024, S E=0.009,95 \%$ CI [0.007, 0.042], $p=.008, B F_{01}=242.8$ (see Figure 3c). However, this difference was small and a Bayesian analysis yielded decisive evidence in favor of the null hypothesis. For participants who scored one standard deviation higher than the mean on need to belong, a one-point increase in apparent extraversion was associated with a 0.68-point increase in liking on our 9-point scale, $b=0.680, S E=0.097,95 \%$ CI [0.469, 0.822], $p<.001$, $B F_{10}=2.05 \times 10^{6}$. For participants who scored one standard deviation below the mean on need to belong, a one-point increase in apparent extraversion was associated with a 0.64-point increase in liking, $b=0.643, S E=0.086,95 \%$ CI [0.496, 0.855], $p<.001, B F_{10}=5.45 \times 10^{6}$.

We again examined whether participants with a more unrestricted sociosexual orientation would show stronger extraversion preferences and whether this association would be stronger for women rating men (vs. men rating women). We estimated a model in which likeability ratings were regressed on apparent extraversion, sociosexual orientation (with larger scores indicating a 
more unrestricted sociosexual orientation $)$, gender $(-0.5=$ women rating men, $0.5=$ men rating women), all two-way interactions, and the three-way interaction. The predicted two-way interaction between apparent extraversion and sociosexual orientation was not significant (with decisive evidence in favor of the null hypothesis), $b=-0.033, S E=0.029,95 \%$ CI [-0.094, 0.030], $p=.253, B F_{01}=876.6$, but the predicted three-way interaction with gender was, $b=-$ $0.118, S E=0.059,95 \%$ CI $[-0.233,0.002], p=.044, B F_{01}=1152$ (see Figure 3e). This time, the pattern was more in line with the original findings, but a Bayesian analysis again yielded decisive evidence in favor of the null hypothesis. We analyzed the interaction between apparent extraversion and sociosexual orientation separately for male and female participants. This showed a marginally significant effect in the predicted direction for female participants (but with decisive evidence in favor of the null hypothesis), $b=0.026, S E=0.014,95 \%$ CI [-0.004, 0.053], $p=.071, B F_{01}=638.7$, and a non-significant effect for male participants (with very strong evidence in favor of the null hypothesis), $b=-0.093, S E=0.059,95 \%$ CI [-0.208, 0.014], $p=.115, B F_{01}=85.22$.

Finally, we found a significant interaction effect between apparent extraversion and participants' level of extraversion (with decisive evidence in favor of the alternative hypothesis), $b=0.054, S E=0.009,95 \%$ CI [0.036, 0.072], $p<.001, B F_{10}=5714$ (see Figure 3d).

Participants who scored one standard deviation higher than the mean on extraversion showed a stronger liking for extraverted-looking individuals, $b=0.737, S E=0.103,95 \%$ CI [0.461, 0.800], $p<.001, B F_{01}=3.48 \times 10^{6}$, than participants who scored one standard deviation below the mean on extraversion, $b=0.632, S E=0.085,95 \%$ CI $[0.513,0.959], p<.001, B F_{10}=4.94 \times$ $10^{6}$. Although the size of the interaction effect was relatively small, it was more than twice as strong as the interaction effect for need to belong. 


\section{Figure 3}

Associations between facial extraversion and likeability ratings for participants scoring low (-1 SD; orange lines) or high (+1 SD; blue lines) on (a) perceived infectability, (b) germ aversion, (c) need to belong, (d) extraversion, and (e) sociosexual orientation (the graph displays results for the predicted interaction effect with participants' gender; Study 2).
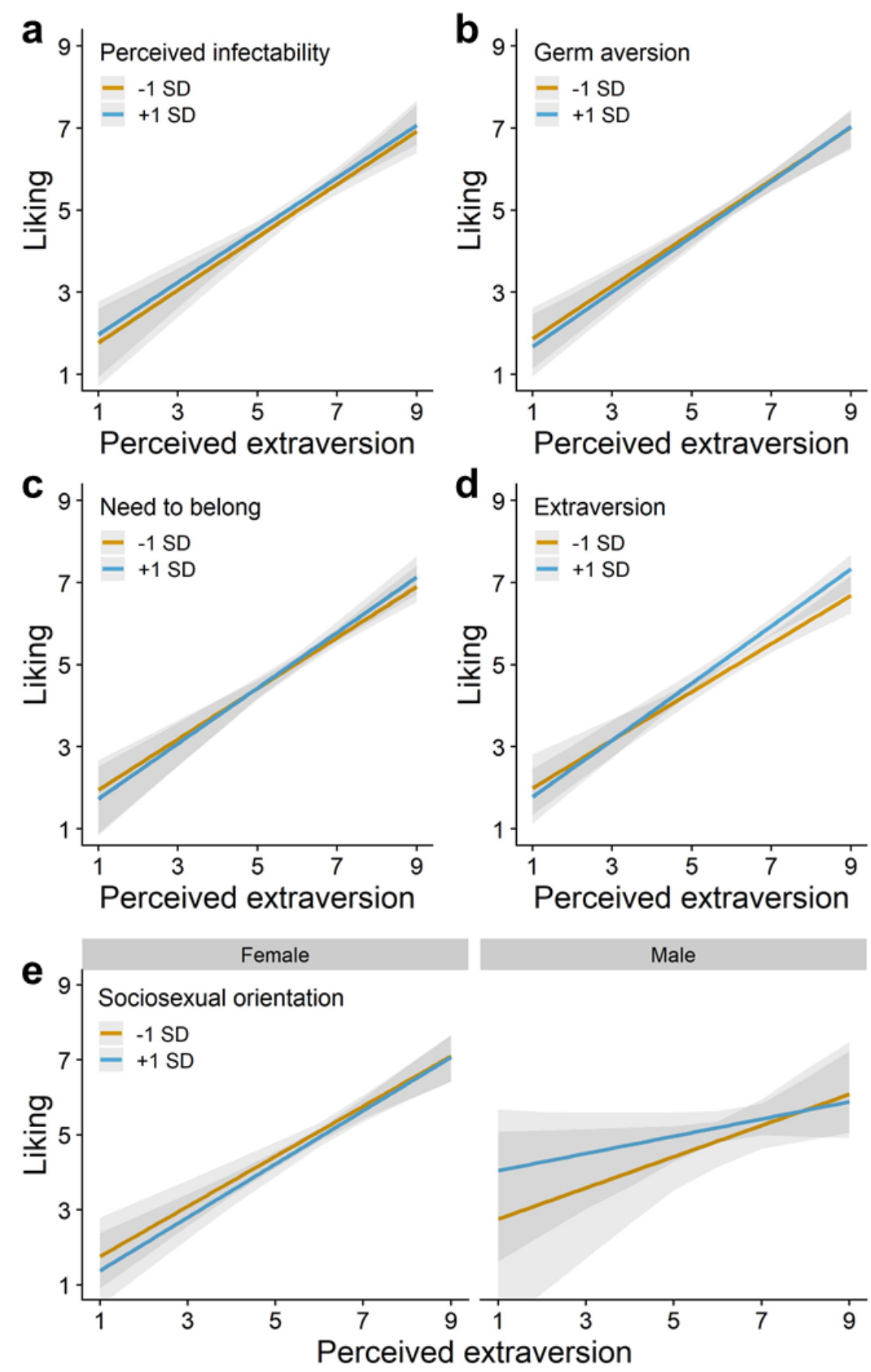

Note. All individual difference measures were $z$-standardized. 


\section{Discussion}

In Study 2, we conducted two additional tests of our hypotheses. We examined the robustness of the original and our previous results by conducting replications (a) using the same study design but a different set of face images and (b) using an alternative, arguably more robust method for measuring participants' impression (DeBruine, 2020; Jones \& Jaeger, 2019) and a third set of face images. In line with the results of our first study, we did not find consistent evidence for associations between $\mathrm{n}$ facial extraversion preferences and individual differences in pathogen concern, need to belong, and sociosexual orientation.

We did find that participants who scored higher on perceived infectability showed a stronger extraversion preference. However, this effect was very small, Bayesian analyses yielded substantial evidence for the null, and the effect only emerged for one stimulus set. The same pattern emerged for the association between need to belong and extraversion preferences. We also found that participants with a more unrestricted sociosexual orientation showed stronger extraversion preferences. However, Bayesian analyses yielded only anecdotal evidence in favor of this effect, and the effect only emerged for one stimulus set. It should also be noted that many of the effects that were significant were just below the significance threshold and would not survive a more conservative correction for multiple tests, which may be appropriate given the large number of tests that we conducted. Thus, overall, we did not find convincing evidence for associations between extraversion preferences and any of the three individual difference measures.

We also examined the role of participants' extraversion and found that participants who scored higher on extraversion showed a stronger preference for extraverted-looking others. While this effect was relatively small, it was supported by Bayesian analyses and emerged for both stimulus samples. Thus, as hypothesized by Sacco and Brown (2018b) but in contrast to their results, which did not yield significant support for their hypothesis, we did find that perceivers form more positive impressions of targets who look like they share the perceiver's level of extraversion.

\section{General Discussion}

How do people form impressions of others based on their facial appearance? Even though recent work has shown that different perceivers reliably form different impressions of targets, the role of individual differences in social perception has remained relatively unexplored (Hehman et 
al., 2019). Perhaps the most comprehensive body of work on this topic was conducted by Brown and Sacco (e.g., 2016b, 2016a, 2017; 2018b). Extending the idea of motivational tradeoffs to social perception (Kenrick et al., 2010; Sacco et al., 2014), they hypothesized that perceivers should form more positive impressions of targets who appear more likely to address their needs. One set of studies tested this idea by examining how various chronic motives of perceivers predict their preferences for individuals varying in apparent extraversion. In the present investigation, we argued that the current evidence in favor of motivational tradeoff effects in social perception is relatively weak and we conducted three preregistered replications of the key findings.

Moving from a relatively close (Study 1, $n=273$ ) to conceptual replications (using two alternative stimulus sets and both the original and an improved study design; Study 2, $n=367$ ), we examined three hypotheses. We tested whether participants who score higher on affiliative needs (Brown \& Sacco, 2017), participants who score lower on pathogen concern (Brown \& Sacco, 2016a), and participants who report a more unrestricted sociosexual orientation (Brown \& Sacco, 2016b) form more positive impressions of extraverted-looking individuals. Across our different tests, we did not find clear support for any of the three hypotheses and Bayesian analyses yielded (usually strong) support for the null. These results emerged consistently across two large samples of participants, three stimulus sets, and two different study designs.

In Study 2, we also examined the role of participants' extraversion. As people tend to like others with similar personalities (Montoya et al., 2008; Youyou et al., 2017), extraverted people may show an increased liking for others who appear extraverted to them. In contrast to an initial study (Sacco \& Brown, 2018b), we did find evidence for such a similarity effect (although the effect size was small). Participants who scored higher on extraversion formed more positive impressions of more extraverted-looking targets. Although the effect was small, it did emerge consistently for both stimulus samples and for frequentist and Bayesian analyses.

\section{Comparison and Integration with Previous Work}

For each of the three primary hypotheses that we examined (testing the role of individual differences in affiliative needs, pathogen concern, and sociosexual orientation), we find broadly consistent null results across our two participant samples and three stimulus sets. What can explain this difference in results compared to the original studies? It is unlikely that the current results are due to low statistical power. Our sample sizes were larger than the sample sizes in the 
original studies. More importantly, Bayesian analyses indicated (usually strong) evidence in favor of for the null hypothesis (rather than weak or anecdotal evidence, which we would expect with underpowered tests).

One salient difference is that the present studies were conducted during the COVID-19 pandemic. For example, it is plausible that the pandemic increased participants' pathogen concern (due to the increased risk of infection and the salience of infectious disease in everyday life) and affiliative needs (due to decreases in social contact resulting from social distancing and isolation). However, it is less clear how this would lead to the observed null results. If there actually is a positive association between affiliative needs and extraversion preferences, then this association should still emerge if an exogenous factor, such as COVID-19, increases everyone's affiliative needs and extraversion preferences. In other words, we would expect that the pandemic increased average scores on some variables of interest, but we would not necessarily expect that this should alter the correlation between two variables. It is possible that the pandemic influenced some variable of interest to such an extent that we do not obtain significant associations due to floor or ceiling effects (e.g., there might be virtually no variation in pathogen concern because everyone scores extremely high on it). This was not the case (see Table 1). Average scores on perceived infectability (Study 1: $M=3.36$, Study 2: $M=3.24$ ) and germ aversion (Study 1: $M=4.07$, Study 2: $M=3.76$ ), which should be most strongly affected by COVID-19, were around or below the midpoint of the seven-point scale. Variation on perceived infectability (Study 1: $S D=1.16$, Study 2: $S D=1.01$ ) and germ aversion (Study 1: $S D=1.17$, Study 2: $S D=1.07$ ) was also similar compared to previous studies (e.g., Duncan et al., 2009).

It should also be noted that, although COVID-19 undoubtedly had a large impact on everyday life, its influence on human psychology may be more nuanced. Others have pointed out that pathogen avoidance mechanisms likely evolved to respond to pathogen threats that are very different from the context of the COVID-19 pandemic (Ackerman et al., 2021). In other words, not every psychological change that we would expect people to display in response to a pathogen threat may emerge in response to the current pandemic. For example, even though several theoretical accounts predict an effect of pathogen threats on political attitudes, a recent largescale investigation found no substantive shift across 84 attitudes in response to the onset of the COVID-19 pandemic (Brandt et al., 2023). Taken together, the considerations outlined above 
highlight that there are no strong reasons to suspect that the present null results were somehow caused by the onset of the COVID-19 pandemic.

In the current studies, we examined correlations between chronic social motives and extraversion preferences. An alternative way to test the role of social motives is by experimentally manipulating them (see, for example, Brown, Medlin, et al., 2019; Brown, Sacco, et al., 2019; Brown \& Sacco, 2016a). Overall, experimental tests seem to converge more with the present (null) findings. In two separate studies, making disease threats salient did not affect participants extraversion preferences (Brown, Medlin, et al., 2019; Brown \& Sacco, 2016a). Socially excluding participants, which increased their need for affiliation, did increase their extraversion preferences, but this effect (surprisingly) only emerged for male participants (Brown, Sacco, et al., 2019). Overall, considering both correlational an experimental approaches, the existing evidence casts doubt on the notion that affiliative needs or pathogen concern are related to preferences for extraverted-looking targets.

\section{Limitations and Future Directions}

One limitation of the current studies is our reliance on Dutch, mostly female, university students. In their original studies, samples of U. S. American participants were recruited from Amazon Mechanical Turk. While this is an important difference between the design of the original studies and our replication studies, it is not clear why the hypotheses tested here would not apply to our samples or why these different samples should yield different results.

Nonetheless, it should be noted that due to our relatively low number of male participants (Study 1: $n=81$, Study $2: n=56$ ), the current studies may have been underpowered to detect predicted sex differences for the association between sociosexual orientation and extraversion preferences (though Bayesian analyses indicated very strong evidence in favor of the null, which somewhat alleviate this concern).

Our results question whether the three individual difference variables studied here meaningfully relate to the positivity of impressions of individuals varying in apparent extraversion, as was suggested in the original investigations. More broadly, the current resultstogether with the mixed correlational and experimental evidence from previous investigations which we highlighted here - show that motivational tradeoff effects in social perception are less robust and less empirically supported than has been suggested before. We do not mean to suggest that the current investigation provides a definitive answer, making future studies on the topic 
unnecessary. Rather, we would encourage future work on this topic that improves on the methodological shortcomings of existing studies.

We see two important developments to improve the robustness of findings on this topic. First, many studies, including the three that we tried to replicate here, relied on the same set of stimuli. To ensure that results are not due to the idiosyncratic set of stimuli that was sampled, future studies should rely on other larger and more diverse stimulus sets (Wells \& Windschitl, 1999). Second, the study design that was used in most previous research on this topic, in which impressions or preferences are measured by showing participants morphed version of the same face in a two-alternative forced-choice design, has been heavily criticized for its poor external validity and tendency to produce false positive results (Bovet et al., 2022; DeBruine, 2020; Jones \& Jaeger, 2019; Satchell et al., 2023). Serial rating tasks, such as the one employed in Study 2, in which participants view unmanipulated faces (with naturalistic variation in, for example, apparent extraversion) should produce more valid results.

Hehman and colleagues' $(2017,2019)$ work suggests that a large amount of variance in first impressions is explained by the interplay between perceiver and target characteristics, but these interactions have received relatively little attention in the literature thus far. Thus, going beyond the characteristics examined the present studies, which may not play a significant role in social perception after all, future work should explore how other characteristics of perceivers influence the impression formation process. For instance, some studies have focused on the cultural background of perceivers (Han et al., 2018; Sofer et al., 2017) to explain differences in social perception.

Finally, future studies should examine the accuracy of face-based extraversion perceptions. The theoretical reasoning underlying the hypotheses examined here rest on the assumption that people can detect how extraverted others are based on their facial appearance. Brown and Sacco (2016b, 2016a, 2017) cite studies that yielded evidence in favor of detection accuracy (Borkenau et al., 2009; Little \& Perrett, 2007), but several other studies yielded null results (Ames et al., 2010; Jones et al., 2012; Shevlin et al., 2003). Thus, it remains unclear if face-based extraversion impressions are at least somewhat accurate and additional data is needed to address this question. 


\section{Conclusion}

How do individual differences shape social perception? Variance component analyses suggest that the interplay between perceiver and target characteristics explains a substantial amount of variance in first impressions. However, these interactions have remained largely unexplored. One body of work tried to explain these interactions though the lens of motivational tradeoffs: Perceivers should form more positive impressions of targets who appear more likely to address salient needs (Brown \& Sacco, 2016a, 2016b, 2017). However, the empirical evidence in favor of motivational tradeoff effects in social perception is relatively weak. Here, we tried to replicate three prior findings, examining the role of three individual difference variables that were hypothesized to influence how people perceive targets varying in apparent extraversion. Across two studies, we used the original stimuli and two additional stimulus sets, and the original study design and an alternative, improved, study design. We did not find evidence for any of the three hypotheses (and Bayesian analyses yielded support in favor of the null hypothesis): Impressions of more or less extraverted-looking targets were not related to individual differences in pathogen concern, need to belong, or sociosexual orientation. We did however find that extraverted participants showed a stronger preference for apparent extraversion in others. Overall, our results call into question the idea that the motivational tradeoffs framework, as operationalized in the current and previous studies, is a valuable lens through which individual differences in social perception can be understood. More importantly, our findings highlight the need for additional research to understand how individual differences shape social perception. 


\section{References}

Ackerman, J. M., Tybur, J. M., \& Blackwell, A. D. (2021). What role does pathogen-avoidance psychology play in pandemics? Trends in Cognitive Sciences, 25(3), 177-186. https://doi.org/10.1016/j.tics.2020.11.008

Aczel, B., Palfi, B., \& Szaszi, B. (2017). Estimating the evidential value of significant results in psychological science. Plos One, 12(8). https://doi.org/10.1371/journal.pone.0182651

Altmejd, A., Dreber, A., Forsell, E., Ho, T., Huber, J., Imai, T., Johannesson, M., Kirchler, M., Nave, G., \& Camerer, C. (2019). Predicting the replicability of social science lab experiments. PloS ONE, 14(12), e0225826. https://doi.org/10.1371/journal.pone.0225826

Ames, D. R., Kammrath, L. K., Suppes, A., \& Bolger, N. (2010). Not so fast: The (not-quitecomplete) dissociation between accuracy and confidence in thin-slice impressions. Personality and Social Psychology Bulletin, 26(2), 264-277. https://doi.org/10.1177/0146167209354519

Ashton, M. C., \& Lee, K. (2009). The HEXACO-60: A short measure of the major dimensions of personality. Journal of Personality Assessment, 91(4), 340-345. https://doi.org/10.1080/00223890902935878

Bainbridge, W. A., Isola, P., Blank, I., \& Oliva, A. (2013). Establishing a database for studying human face photograph memory. In N. Miyake, D. Peebles, \& R. P. Coopers (Eds.), Proceedings of the 34th Annual Conference of the Cognitive Science Society (pp. 13021307). Cognitive Science Society.

Bainbridge, W. A., Isola, P., \& Oliva, A. (2013). The intrinsic memorability of face photographs. Journal of Experimental Psychology: General, 142(4), 1323-1334. https://doi.org/10.1037/a0033872

Bates, D., Mächler, M., Bolker, B., \& Walker, S. (2015). Fitting linear mixed-effects models using lme4. Journal of Statistical Software, 67(1). https://doi.org/10.18637/jss.v067.i01

Baumeister, R. F., \& Leary, M. R. (1995). The need to belong: Desire for interpersonal attachments as a fundamental human motivation. Psychological Bulletin, 117(3), 497529. https://doi.org/10.1037/0033-2909.117.3.497

Borkenau, P., Brecke, S., Möttig, C., \& Paelecke, M. (2009). Extraversion is accurately perceived after a 50-ms exposure to a face. Journal of Research in Personality, 43(4), 703-706. https://doi.org/10.1016/j.jrp.2009.03.007 
Bovet, J., Tognetti, A., \& Pollet, T. V. (2022). Methodological issues when using face prototypes: A case study on the Faceaurus dataset. Evolutionary Human Sciences, 4, e48.

Brandt, M. J., Vallabha, S., \& Turner-Zwinkels, F. M. (2023). The onset of the COVID-19 pandemic made people feel threatened, but had a limited impact on political attitudes [Preprint]. PsyArXiv. https://doi.org/10.31234/osf.io/8n52q

Brown, M., Medlin, M. M., Sacco, D. F., \& Young, S. G. (2019). Facing competing motives: Testing for motivational tradeoffs in affiliative and pathogen-avoidant motives via extraverted face preferences. Evolutionary Psychological Science, 5(4), 440-446. https://doi.org/10.1007/s40806-019-00200-5

Brown, M., \& Sacco, D. F. (2016a). Avoiding extraverts: Pathogen concern downregulates preferences for extraverted faces. Evolutionary Psychological Science, 2(4), 278-286. https://doi.org/10.1007/s40806-016-0064-6

Brown, M., \& Sacco, D. F. (2016b). Unrestricted sociosexuality predicts preferences for extraverted male faces. Personality and Individual Differences, 108, 123-127. https://doi.org/10.1007/978-3-319-12616-6

Brown, M., \& Sacco, D. F. (2017). Greater need to belong predicts a stronger preference for extraverted faces. Personality and Individual Differences, 104, 220-223. https://doi.org/10.1016/j.paid.2016.08.012

Brown, M., Sacco, D. F., \& Medlin, M. M. (2019). Approaching extraverts: Socially excluded men prefer extraverted faces. Personality and Individual Differences, 137, 198-203. https://doi.org/10.1016/j.paid.2018.09.007

DeBruine, L. M. (2020). Composite images. https://debruine.github.io/posts/composite-images/

Duarte, J., Siegel, S., \& Young, L. (2012). Trust and credit: The role of appearance in peer-topeer lending. Review of Financial Studies, 25(8), 2455-2483. https://doi.org/10.1093/rfs/hhs071

Duncan, L. A., Schaller, M., \& Park, J. H. (2009). Perceived vulnerability to disease: Development and validation of a 15-item self-report instrument. Personality and Individual Differences, 47(6), 541-546. https://doi.org/10.1016/j.paid.2009.05.001

Gomulya, D., Wong, E. M., Ormiston, M. E., \& Boeker, W. (2017). The role of facial appearance on CEO selection after firm misconduct. Journal of Applied Psychology, 102(4), 617-635. http://dx.doi.org/10.1037/ap10000172 
Han, C., Wang, H., Hahn, A. C., Fisher, C. I., Fasolt, V., Morrison, D. K., Lee, A. J., Iris, J., DeBruine, L. M., \& Jones, B. C. (2018). Cultural differences in preferences for facial coloration. Evolution and Human Behavior, 2, 154-159. https://doi.org/10.1016/j.evolhumbehav.2017.11.005

Hehman, E., Stolier, R. M., Freeman, J. B., Flake, J. K., \& Xie, S. Y. (2019). Toward a comprehensive model of face impressions: What we know, what we do not, and paths forward. Social and Personality Psychology Compass, 13(2), 1-16. https://doi.org/10.1111/spc3.12431

Hehman, E., Sutherland, C. A. M., Flake, J. K., \& Slepian, M. L. (2017). The unique contributions of perceiver and target characteristics in person perception. Journal of Personality and Social Psychology, 113(4), 513-529. https://doi.org/10.1037/pspa0000090

Holtzman, N. S. (2011). Facing a psychopath: Detecting the dark triad from emotionally-neutral faces, using prototypes from the Personality Faceaurus. Journal of Research in Personality, 45(6), 648-654. https://doi.org/10.1016/j.jrp.2011.09.002

Huang, J. Y., \& Bargh, J. A. (2014). The Selfish Goal: Autonomously operating motivational structures as the proximate cause of human judgment and behavior. Behavioral and Brain Sciences, 38(01), 121-135. https://doi.org/10.1017/S0140525X13000290

Jaeger, B., Todorov, A. T., Evans, A. M., \& van Beest, I. (2020). Can we reduce facial biases? Persistent effects of facial trustworthiness on sentencing decisions. Journal of Experimental Social Psychology, 90, 104004. https://doi.org/10.1016/j.jesp.2020.104004

Jaeger, B., Wagemans, F. M. A., Evans, A. M., \& van Beest, I. (2018). Effects of facial skin smoothness and blemishes on trait impressions. Perception, 47(6), 608-625. https://doi.org/10.1177/0301006618767258

Jeffreys, H. (1961). Theory of Probability. Oxford University Press.

Jones, A. L., \& Jaeger, B. (2019). Biological bases of beauty revisited: The effect of symmetry, averageness, and sexual dimorphism on female facial attractiveness. Symmetry, 11(2). https://doi.org/10.3390/SYM11020279 
Jones, A. L., Kramer, R. S. S., \& Ward, R. (2012). Signals of personality and health: The contributions of facial shape, skin texture, and viewing angle. Journal of Experimental Psychology: Human Perception and Performance, 38(6), 1353-1361. https://doi.org/10.1037/a0027078

Kenrick, D. T., Griskevicius, V., Neuberg, S. L., \& Schaller, M. (2010). Renovating the pyramid of needs: Contemporary extensions built upon ancient foundations. Perspectives on Psychological Science, 5(3), 292-314. https://doi.org/10.1177/1745691610369469

Klapper, A., Dotsch, R., van Rooij, I., \& Wigboldus, D. H. J. (2016). Do we spontaneously form stable trustworthiness impressions from facial appearance? Journal of Personality and Social Psychology, 111(5), 655-664. https://doi.org/10.1037/pspa0000062

Kuznetsova, A., Brockhoff, P. B., \& Christensen, R. H. B. (2016). lmerTest: Tests in linear mixed effects models.

Leary, M. R., Kelly, K. M., Cottrell, C. A., \& Lisa, S. (2013). Construct validity of the need to belong scale: Mapping the nomological network. Psychological Assessment, 95(6), 610624. https://doi.org/10.1080/00223891.2013.819511

LeBel, E. P., Vanpaemel, W., Cheung, I., \& Campbell, L. (2019). A brief guide to evaluate replications. Meta-Psychology, 3(August 2018). https://doi.org/10.15626/mp.2018.843

Ling, L., Luo, D., \& She, G. (2019). Judging a book by its cover: The influence of physical attractiveness on the promotion of regional leaders. Journal of Economic Behavior and Organization, 158, 1-14. https://doi.org/10.1016/j.jebo.2019.01.005

Little, A. C., \& Perrett, D. I. (2007). Using composite images to assess accuracy in personality attribution to faces. British Journal of Psychology, 98(1), 111-126. https://doi.org/10.1348/000712606X109648

Montoya, R. M., Horton, R. S., \& Kirchner, J. (2008). Is actual similarity necessary for attraction? A meta-analysis of actual and perceived similarity. In Journal of Social and Personal Relationships (Vol. 25, Issue 6). https://doi.org/10.1177/0265407508096700

Nettle, D. (2005). An evolutionary approach to the extraversion continuum. Evolution and Human Behavior, 26(4), 363-373. https://doi.org/10.1016/j.evolhumbehav.2004.12.004

Open Science Collaboration. (2015). Estimating the reproducibility of psychological science. Science, 349(6251). https://doi.org/10.1126/science.aac4716 
Penke, L., \& Asendorpf, J. B. (2008). Beyond global sociosexual orientations: A more differentiated look at sociosexuality and its effects on courtship and romantic relationships. Journal of Personality and Social Psychology, 95(5), 1113-1135. https://doi.org/10.1037/0022-3514.95.5.1113

Pollet, T. V., Roberts, S. G. B., \& Dunbar, R. I. M. (2011). Extraverts have larger social network layers but do not feel emotionally closer to individuals at any layer. Journal of Individual Differences, 32(3), 161-169. https://doi.org/10.1027/1614-0001/a000048

R Core Team. (2021). R: A language and environment for statistical computing. R Foundation for Statistical Computing, Vienna, Austria. https://www.r-project.org/

Sacco, D. F., \& Brown, M. (2018a). Preferences for facially communicated big five personality traits and their relation to self-reported big five personality. Personality and Individual Differences, 134(June), 195-200. https://doi.org/10.1016/j.paid.2018.06.024

Sacco, D. F., \& Brown, M. (2018b). The face of personality: Adaptive inferences from facial cues are moderated by perceiver personality and motives. Social and Personality Psychology Compass, 12(8), 1-15. https://doi.org/10.1111/spc3.12410

Sacco, D. F., Young, S. G., \& Hugenberg, K. (2014). Balancing competing motives: Adaptive Trade-Offs Are Necessary to Satisfy Disease Avoidance and Interpersonal Affiliation Goals. Personality and Social Psychology Bulletin, 40(12), 1611-1623. https://doi.org/10.1177/0146167214552790

Said, C. P., Sebe, N., \& Todorov, A. (2009). Structural resemblance to emotional expressions predicts evaluation of emotionally neutral faces. Emotion, 9(2), 260-264. https://doi.org/10.1037/a0014681

Satchell, L., Jaeger, B., Jones, A. L., Lopez, B., \& Schild, C. (2023). Beyond reliability in first impressions research: Considering validity and the need to "mix it up with folks." Social Psychological Bulletin. https://doi.org/10.31234/osf.io/4gk6b

Schaller, M., \& Park, J. H. (2011). The behavioral immune system (and why it matters). Current Directions in Psychological Science, 20(2), 99-103. https://doi.org/10.1177/0963721411402596 
Shevlin, M., Walker, S., Davies, M. N. O., Banyard, P., \& Lewis, C. A. (2003). Can you judge a book by its cover? Evidence of self-stranger agreement on personality at zero acquaintance. Personality and Individual Differences, 35(6), 1373-1383. https://doi.org/10.1016/S0191-8869(02)00356-2

Simmons, J. P., Nelson, L. D., \& Simonsohn, U. (2011). False-positive psychology: Undisclosed flexibility in data collection and analysis allows presenting anything as significant. Psychological Science, 22(11), 1359-1366. https://doi.org/10.1177/0956797611417632

Sofer, C., Dotsch, R., Oikawa, M., Oikawa, H., Wigboldus, D. H. J., \& Todorov, A. (2017). For your local eyes only: Culture-specific face typicality influences perceptions of trustworthiness. Perception, 46(8), 914-928. https://doi.org/10.1177/0301006617691786

Stirrat, M., \& Perrett, D. I. (2010). Valid facial cues to cooperation and trust: Male facial width and trustworthiness. Psychological Science, 21(3), 349-354. https://doi.org/10.1177/0956797610362647

Tierney, W., Hardy, J. H., Ebersole, C. R., Leavitt, K., Viganola, D., Clemente, E. G., Gordon, M., Dreber, A., Johannesson, M., Pfeiffer, T., Uhlmann, E. L., Abraham, A. T., Adamkovic, M., Adam-Troian, J., Anand, R., Arbeau, K. J., Awtrey, E. C., Azar, O. H., Bahník, Š., ... Øverup, C. S. (2020). Creative destruction in science. Organizational Behavior and Human Decision Processes, 161, 291-309. https://doi.org/10.1016/j.obhdp.2020.07.002

Todorov, A., Olivola, C. Y., Dotsch, R., \& Mende-Siedlecki, P. (2015). Social attributions from faces: Determinants, consequences, accuracy, and functional significance. Annual Review of Psychology, 66(1), 519-545. https://doi.org/10.1146/annurev-psych-113011-143831

Tybur, J. M., Frankenhuis, W. E., \& Pollet, T. V. (2014). Behavioral immune system methods: Surveying the present to shape the future. Evolutionary Behavioral Sciences, 8(4), 274283. https://doi.org/10.1037/ebs0000017

Tybur, J. M., \& Lieberman, D. (2016). Human pathogen avoidance adaptations. Current Opinion in Psychology, 7, 6-11. https://doi.org/10.1016/j.copsyc.2015.06.005

Tybur, J. M., Lieberman, D., Fan, L., Kupfer, T. R., \& de Vries, R. E. (2020). Behavioral immune trade-offs: Interpersonal value relaxes social pathogen avoidance. Psychological Science, 31(10), 1211-1221. https://doi.org/10.1177/0956797620960011 
Wagenmakers, E.-J. (2007). A practical solution to the pervasive problems of $\mathrm{p}$ values. Psychonomic Bulletin \& Review, 14(5), 779-804. https://doi.org/10.3758/BF03194105

Walker, M., Schönborn, S., Greifeneder, R., \& Vetter, T. (2018). The Basel Face Database: A validated set of photographs reflecting systematic differences in big two and big five personality dimensions. PLoS ONE, 13(3), e0193190.

https://doi.org/10.1371/journal.pone.0193190

Walker, M., \& Vetter, T. (2016). Changing the personality of a face: Perceived Big Two and Big Five personality factors modeled in real photographs. Journal of Personality and Social Psychology, 110(4), 609-624. https://doi.org/10.1037/pspp0000064

Wells, G. L., \& Windschitl, P. D. (1999). Stimulus Sampling and Social Psychological Experimentation. Personality and Social Psychology Bulletin, 25(9), 1115-1125. https://doi.org/10.1177/01461672992512005

Willis, J., \& Todorov, A. (2006). First impressions: Making up your mind after a 100-ms exposure to a face. Psychological Science, 17(7), 592-598. https://doi.org/10.1111/j.1467-9280.2006.01750.x

Xie, S. Y., Flake, J. K., \& Hehman, E. (2019). Perceiver and target characteristics contribute to impression formation differently across race and gender. Journal of Personality and Social Psychology, 117(2), 364-385. https://doi.org/10.1037/pspi0000160

Young, S. G., Brown, M., \& Sacco, D. F. (2021). Using psychological science to support social distancing: Tradeoffs between affiliation and disease-avoidance motivations. Social and Personality Psychology Compass. https://doi.org/10.1111/spc3.12597

Youyou, W., Stillwell, D., Schwartz, H. A., \& Kosinski, M. (2017). Birds of a feather do flock together: Behavior-based personality-assessment method reveals personality similarity among couples and friends. Psychological Science, 28(3), 276-284. https://doi.org/10.1177/0956797616678187

Zebrowitz, L. A., \& McDonald, S. M. (1991). The impact of litigants' baby-facedness and attractiveness on adjudications in small claims courts. Law and Human Behavior, 15(6), 603-623. https://doi.org/10.1007/BF01065855 